\section{CXCR2 antagonists in breast cancer}

\section{By Lauren Martz, Staff Writer}

A team at the Memorial Sloan-Kettering Cancer Center has shown that CXC chemokine receptor 2 antagonists sensitized tumors to chemotherapy in mouse models of metastatic breast cancer. ${ }^{1}$ The team is working on additional preclinical studies with the intention of moving the antagonists into clinical trials for breast cancer.

Resistance to chemotherapy often occurs in breast cancer as a result of a small percentage of cancer cells that develop a survival advantage and show a more aggressive, metastatic phenotype.

The challenge has been identifying the factors that confer the survival advantage and figuring out how to target them in combination with chemotherapy to prevent resistance, tumor relapse and metastasis.

In 2005, the Sloan-Kettering team, led by Joan Massagué, found that expression of chemokine CXC motif ligand 1 (CXCL1; GRO; MGSA) and CXCL2 (MIP2) on breast cancer cells was associated with risk of relapse and metastasis to the lungs. ${ }^{2}$

Building on that work, the group set out to determine the mechanism that linked CXC ligand expression on tumors to chemotherapeutic resistance, with the hope of identifying molecular targets to prevent resistance.

Massagué is chairman of the Cancer Biology and Genetics Program and director of the Metastasis Research Center at Memorial Sloan-Kettering. He is also a Howard Hughes Medical Institute investigator. The team included researchers from Cancer Research UK.

The team found that CXCL1 and CXCL2 were amplified in $7.5 \%$ of human primary breast cancer tissues tested and 19.9\% of metastases, suggesting both chemokines were associated with invasiveness. In mouse xenograft models of metastatic breast cancer, small hairpin RNA against CXCL1 and CXCL2 decreased mammary tumor growth and metastasis compared with shRNA control.

Although the levels of CXCL1 and CXCL2 were elevated in the breast cancer tissue, the levels of their receptors, including CXC chemokine receptor 2 (CXCR2; IL8RB), were low. That suggested the prosurvival effects of the ligands might be the result of their actions on tissues surrounding the tumor.

Thus, the team set out to determine whether the ligands were altering the activity of cells within the tumor microenvironment.

Indeed, in the same mouse models, CXCL1 and CXCL2 knockdown decreased levels of complement receptor 3 (Cr3; Cd11b)+/lymphocyte antigen 6 complex locus G (Ly6g; Gr1) ${ }^{+}$myeloid cells, which express higher levels of CXCR2 than other stromal cells, in the tumor microenvironment.

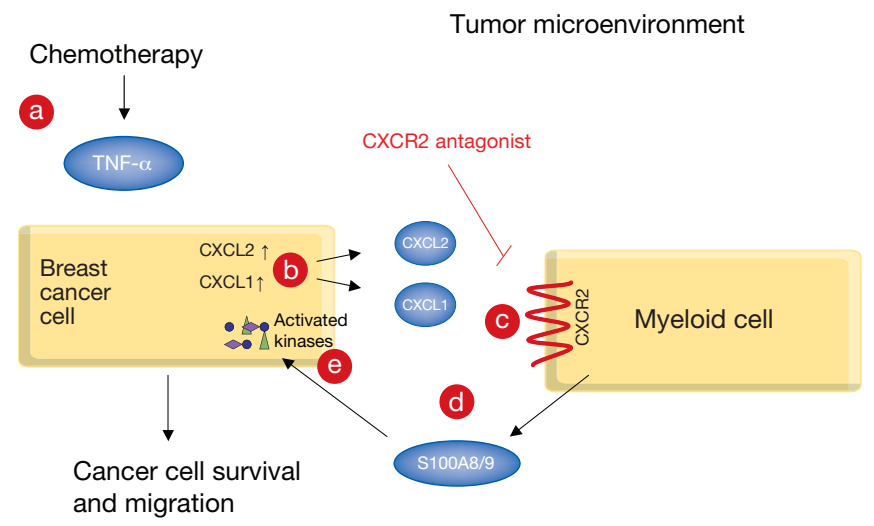

Figure 1. Breast cancer survival network. Chemotherapy results in a burst of paracrine factors near breast tumors that upregulate survival pathways in the cancer cells, causing chemotherapeutic resistance and metastasis. The process begins when chemotherapy induces the release of tumor necrosis factor- $\alpha$ (TNF- $\alpha$ ) [a] from stromal cells in the breast, including endothelial cells. As a result, chemokine CXC motif ligand 1 (CXCL1; GRO; MGSA) and CXCL2 (MIP2) are upregulated in breast cancer cells [b] by mechanisms including TNF- $\alpha$ release. The release of CXCL1 and CXCL2 attracts CXC chemokine receptor 2 (CXCR2; IL8RB)expressing stromal cells from the tumor microenvironment to the tumor. Specifically, CXCR2-expressing, complement receptor 3 (CR3; CD11b)+/lymphocyte antigen 6 complex locus G (LY6G; GR1)+ myeloid cells accumulate near the tumor [c]. The myeloid cells release paracrine factors including $S 100$ calcium binding protein A8 (S100A8; calgranulin A; MRP8) and S100A9 (calgranulin B; MRP14) [d], which activate kinases such as MAP kinase 3 (MAPK3; ERK-1), MAPK1 (ERK-2), MAPK and ribosomal protein S6 kinase $70 \mathrm{kDa}$ polypeptide 1 (RPS6KB1; S6K1) on the breast cancer cells [e]. Activation of the cancer-associated kinases promotes cancer cell survival and metastasis.

CXCR2 antagonists prevent CXCR2-expressing myeloid cells from homing to cancer cells, blocking release of S100A8 and S100A9 and subsequent kinase signaling that promotes cancer survival. Blocking CXCR2 could reduce chemotherapy resistance and breast cancer metastasis.

Moreover, coculture of the tumor and $\mathrm{Cd} 11 \mathrm{~b}^{+} / \mathrm{Gr}^{+}$myeloid cells prevented the tumor cells from undergoing chemotherapy-induced apoptosis. That suggested the $\mathrm{Cd} 11 \mathrm{~b}^{+} / \mathrm{Gr}^{+}$myeloid cells were mediating the chemotherapy resistance of the tumor cells.

A subsequent series of experiments revealed a mechanism whereby CXCL1 and CXCL2 bound CXCR 2 on nearby Cd11 $\mathrm{b}^{+} / \mathrm{Gr} 1^{+}$myeloid cells, which triggered the secretion of two survival factors, $\mathrm{S} 100$ calcium binding protein A8 (S100a8; calgranulin A; Mrp8) and S100a9 (calgranulin B; Mrp14). Those survival factors then acted on the tumor to promote survival in the presence of chemotherapy (see Figure 1, "Breast cancer survival network").

Finally, the group tested whether it might be possible to block the entire 


\section{ANALYSIS}

\section{TARGETS \& MECHANISMS}

mechanism using CXCR2 antagonists in combination with chemotherapy.

In a mouse breast cancer xenograft model, doxorubicin plus a CXCR2 antagonist lowered metastatic burden and tumor growth better than doxorubicin alone.

The results were published in Cell.

"The tumor-promoting effects of the tumor microenvironment have been gaining attention, and the CXCL/CXCR2 axis seems to be one of the key signals in the tumor microenvironment," Ijichi Hideaki told SciBX. $\mathrm{He}$ is a research associate in the Department of Gastroenterology at The University of Tokyo Hospital.

"Combination of conventional chemoreagents, which target tumor cells directly, and modulators of the tumor microenvironment might be a promising strategy for synergistic treatment of cancer," he added.

\section{Next steps}

Before CXCR2 antagonists can be tested in combination with chemotherapeutics in the clinic, researchers agree that extensive preclinical evaluation of efficacy will be required. It will also be necessary to address a few potential adverse effects.

The Sloan-Kettering study "used mostly xenograft models, and they did not examine CXCR2-antagonizing effects on genetically engineered models," said Hideaki. "If they could also see consistent results as well as prolonged overall survival using these models, it would strengthen their findings."

Indeed, Massagué told SciBX his team's next steps include extensive preclinical analysis with different CXCR2 inhibitors and chemotherapeutics for different durations. "Patient-derived grafts and transgenic models need to be tested side by side with xenografts to understand the full gamut of efficacy of these CXCR2 inhibitors in reducing breast metastasis," he said.

These studies are underway.

Potential side effects need to be evaluated as well, said Hideaki.

"Since CXCR2 is known to be involved in the host resistance to bacterial and fungal infections and the wound healing response, antagonizing CXCR2 might cause certain disadvantages," he said. "This should be carefully investigated in vivo using multiple genetically engineered cancer models to clarify not only the therapeutic effects but also certain adverse effects."

He added that humans have an additional CXCR1 and CXCR2 ligand that mice lack, IL-8 (CXCL8), which is involved in many aspects of different diseases. Because most in vivo functional evidence of the CXCR2antagonism effect was shown using mouse models, the researchers should be careful about this difference between humans and mice, he said.

At least two CXCR2 antagonists have already been evaluated in clinical safety studies, although according to Hideaki "no trials have been performed in cancers."

CXCR2 antagonists in the clinic include AstraZeneca plc's AZD5069, which is in Phase II testing to treat chronic obstructive pulmonary disease (COPD), and Dompe Farmaceutici S.p.A.'s Reparixin, which is in Phase II testing for graft and renal transplant rejection. The companies declined to comment.

\section{Markers}

In addition to the potential therapeutic applications of the findings, the team thinks the results have important implications for breast cancer diagnosis and prognosis.

Massagué told SciBX his team has confirmed a strong association between S100A 8 and S100A9 and resistance to perioperative chemotherapy. "This could lead to useful markers of resistance and relapse in the future."

He added, "Since S100A8 and S100A9 are easy to detect in serum and tissues, these markers might also be useful for developing clinical assays in the future."

Massagué said Sloan-Kettering has filed a provisional U.S. patent application covering the work, and the IP is available for licensing.

Martz, L. SciBX 5(31); doi:10.1038/scibx.2012.807

Published online Aug. 9, 2012

\section{REFERENCES}

1. Acharyya, S. et al. Cell; published online July 6, 2012; doi:10.1016/j.cell.2012.04.042

Contact: Joan Massagué, Memorial Sloan-Kettering Cancer Center, New York, N.Y. e-mail: massaguj@mskcc.org

2. Minn, A.J. et al. Nature 436, 518-524 (2005)

\section{COMPANIES AND INSTITUTIONS MENTIONED}

AstraZeneca plc (LSE:AZN; NYSE:AZN), London U.K.

Cancer Research UK, London, U.K.

Dompe Farmaceutici S.p.A., Milan, Italy

Howard Hughes Medical Institute, Chevy Chase, Md.

Memorial Sloan-Kettering Cancer Center, New York, N.Y. The University of Tokyo Hospital, Tokyo, Japan 